\title{
Metodologia integrada de acompanhamento de políticas públicas e situação de saúde: o SISAP-Idoso
}

\author{
An integrated methodology for Monitoring and Evaluating \\ the Public Policies and Health Situation of the Elderly: SISAP-Idoso
}

Dalia Elena Romero ${ }^{1}$

Débora Castanheira ${ }^{2}$

Aline Pinto Marques ${ }^{1}$

Jessica Muzy ${ }^{1}$

Leticia Sabbadini ${ }^{1}$

Raulino Sabino da Silva ${ }^{1}$
${ }^{1}$ Instituto de Comunicação e Informação Científica e Tecnológica em Saúde, Fiocruz. Av. Brasil 4.365/ Pavilhão Haity Moussatché, Manguinhos. 21040900 Rio de Janeiro RJ Brasil.dalia.romero@ icict.fiocruz.br

${ }^{2}$ Instituto de Estudos Sociais e Políticos,

Universidade do Estado do Rio de Janeiro. Rio de Janeiro RJ Brasil.

\begin{abstract}
This article examines the strategy for construction of a System of Indicators for Monitoring the Public Policies and Health Situation of the Elderly (SISAP-Idoso). The System seeks to assess the production of health information with the political/institutional decision, serving as a management tool in the health of the elderly. Its focus is to meet the need of the Unified Health System municipal managers for information for planning and controlling health programs. The paper presents the methods used in the creation of the Conceptual Matrix for Health Dimensions and Conceptual Matrix for Monitoring and Evaluation of Policies. The first systematizes a panel of indicators to diagnose the health condition of the elderly, a procedure without which one cannot properly plan health actions. The second selects indicators to monitor implementation and evaluate the results of health policies and programs for the elderly. The System became an important tool to support the formulation and monitoring of health programs, particularly at the local level. Moreover, it is a powerful tool of social control and population empowerment. The full incorporation of the System depends on the consolidation of information and indicators as parts of the policy process.
\end{abstract}

Key words Health management, Health evaluation, Health policy, Indicators, Information systems
Resumo O artigo trata da estratégia de construção do Sistema de Indicadores de Saúde e Acompanhamento de Políticas do Idoso (SISAP-Idoso). O Sistema pretende articular a produção de informações em saúde com a decisão político-institucional, de modo a servir como instrumento de gestão em saúde do idoso. Seu foco é suprir a necessidade de informações para o planejamento e o controle de politicas e programas dos gestores municipais do Sistema Único de Saúde (SUS). Apresentam-se as metodologias de criação da Matriz Conceitual por Dimensões de Saúde e da Matriz Conceitual para Acompanhamento de Políticas e Programas. A primeira sistematiza um painel de indicadores para o diagnóstico da situação de saúde da população idosa, procedimento sem o qual não é possível planejar adequadamente ações em saúde. A segunda seleciona indicadores para monitorar a implementação e avaliar os resultados de políticas e programas de saúde do idoso. $O$ Sistema se mostra uma importante ferramenta de suporte da formulação e acompanhamento de programas de saúde, principalmente no nivel municipal. Além disso, é uma poderosa ferramenta de controle social e de empoderamento da população. A incorporação plena do SISAP-Idoso depende da consolidação do uso de informação e de indicadores no processo de políticas públicas.

Palavras-chave Gestão em saúde, Avaliação em saúde, Política de saúde, Indicadores, Sistemas de informação 


\section{Introdução}

Um problema subjacente à consolidação das políticas de saúde do idoso no Brasil é a falta de mecanismos de monitoramento da implementação e avaliação dos resultados dos investimentos neste setor. Apesar de ser considerado pioneiro na criação de leis e políticas públicas que visam à proteção social da pessoa idosa, o país tem dificuldades em monitorar e avaliar estas medidas em razão da ausência de indicadores sensíveis.

Em que pese as recentes iniciativas do Ministério da Saúde, os avanços, na maior parte dos casos, constituíram-se em iniciativas pontuais, mediante pesquisas específicas, estudos de casos. Mesmo quando há produção regular de dados, não há equivalente regularidade de análise orientada para as necessidades da gestão. O próprio Ministério da Saúde ${ }^{1}$ identifica alguns dos limites dessas iniciativas: 1) a ausência ou insuficiência da explicitação das diretrizes políticas e da definição estratégica que orientam a avaliação; 2) a fragmentação e a decorrente diversidade de orientações que presidem os processos de avaliação, impedindo que possam ser úteis a uma ação coordenada; 3) a ênfase em variáveis relacionadas a processo; e, 4) avaliações de resultados pontuais e/ou espacialmente restritas ${ }^{2}$.

O SISAP-Idoso - Sistema de Indicadores para Acompanhamento de Políticas de Saúde do Idoso (https://sisapidoso.icict.fiocruz.br/) foi lançado em 2011 pelo Laboratório de Informação em Saúde do Instituto de Comunicação e Informação Científica e Tecnológica em Saúde (LIS-ICICT/Fiocruz) para suprir esta dificuldade. O Sistema foi criado inicialmente como Monitor Idoso em 2005 como uma iniciativa do ICICT, financiada pelo Programa de Indução à Pesquisa e Desenvolvimento Tecnológico (PIPDT). Em 2007 foi iniciada uma parceria do ICICT com a Coordenação de Saúde da Pessoa Idosa do Ministério da Saúde, transformando o Sistema em um produto compartilhado entre Fiocruz e Ministério da Saúde.

O SISAP-Idoso compreende um sistema de consulta de indicadores pela internet, a nível federal, estadual e municipal, sobre a saúde do idoso. A finalidade é oferecer uma ferramenta para a gestão do SUS que permita ao mesmo tempo conhecer a situação de saúde da população idosa e estabelecer processos contínuos de acompanhamento (ou seja, monitoramento e avaliação - M\&A). A proposta do sistema é fornecer aos gestores e à sociedade como um todo dados úteis à formulação de políticas e ações de prevenção, além de contribuir na melhoria da qualidade da assistência à saúde e da informação gerada nos níveis básicos de atenção do SUS.

O objetivo deste artigo é apresentar a estratégia de construção do SISAP-Idoso. Com isso, pretendemos demonstrar como o Sistema pode ser utilizado para o desenvolvimento de funções como planejamento, programação, regulação, controle e avaliação dos programas e políticas de saúde do idoso.

$\mathrm{O}$ artigo será dividido em três partes. $\mathrm{Na}$ primeira parte será apresentada a metodologia de criação da Matriz Conceitual por Dimensões de Saúde e, na segunda, a da Matriz Conceitual para Acompanhamento de Políticas e Programas. Por último, apresentamos as considerações finais quanto à usabilidade, desafios e perspectivas. Espera-se que o sistema seja útil para realizar o diagnóstico da situação de saúde da população idosa e para o acompanhamento de políticas públicas nas diferentes fases de seu ciclo.

\section{A lógica de estruturação dos indicadores no SISAP-Idoso}

Desde a década de 1980, gestores no campo da saúde, em geral, e da saúde do idoso, em particular, deparam-se frente ao desafio de encontrar soluções para a organização e o funcionamento dos sistemas de saúde face às mudanças demográficas e epidemiológicas, ao aumento das despesas com assistência médico-hospitalar e seus efeitos sobre o bem-estar da população e o progresso contínuo das tecnologias médicas. Estes desafios só podem ser enfrentados com o uso de informação adequada e oportuna para o planejamento e o acompanhamento de ações de saúde.

No entanto, como indica a pesquisa realizada por Cohn et al. ${ }^{3}$, a falta de familiaridade dos gestores municipais com os sistemas de informação em saúde e a falta de conhecimento para manipular e analisar os dados disponíveis impedem que a análise periódica da situação de saúde sirva de insumo para a elaboração e o acompanhamento de planos e programas de saúde. Desta forma, a aproximação entre os gestores de saúde e os sistemas de informações configura-se fator importante para que indicadores de saúde sejam incorporados no processo de decisão.

A criação de sistemas de diagnóstico e acompanhamento do sistema de saúde ganha destaque. O SISAP-Idoso foi criado para responder a essa demanda. O M\&A surge como ferramenta gerencial poderosa, fornecendo aos formuladores de políticas públicas e aos gestores de programas 
condições para aumentar a eficiência, a efetividade e a equidade dos recursos aplicados em programas de saúde ${ }^{4}$.

O sistema tem como diretriz o estabelecimento de mecanismos que favoreçam a divulgação de informações sobre o processo de envelhecimento para a população em geral, mas em especial para aquela idosa e para os gestores do sistema de saúde, tal qual estabelecido no Estatuto do Idoso (art $3^{\circ}$, VII) e na Política Nacional do Idoso (art. $3^{\circ}$, II e art. $4^{\circ}$, VII). Grande parte da inovação do SISAP -Idoso vem da combinação de duas lógicas independentes, mas complementares de estruturação dos indicadores. A primeira delas é fruto de uma adaptação da Matriz de Dimensões da Avaliação do Sistema de Saúde no Brasil, desenvolvida em 2003 pelo Projeto de Avaliação de Desempenho de Sistemas de Saúde (PROADESS $)^{5,6}$. O objetivo desta matriz é fornecer aos gestores e à população uma ferramenta para realizar a análise de situação de saúde da população idosa, procedimento sem o qual não é possível planejar adequadamente ações de saúde.

A segunda, chamada Matriz Conceitual para o Acompanhamento de Políticas e Programas, é uma inovação do SISAP-Idoso que tem como objetivo permitir o acompanhamento (monitoramento da implementação e avaliação de resultados) de dispositivos específicos de políticas e programas de saúde da pessoa idosa através de indicadores selecionados. Sua função é subsidiar a formulação e o acompanhamento de políticas e programas de saúde do idoso para encorajar a utilização apropriada dos recursos públicos, desestimular o uso inadequado de procedimentos, serviços e tecnologias e promover a prestação de serviços de saúde com maior eficiência, eficácia e equidade (Figura 1).

A elaboração do SISAP-Idoso foi a construção de um marco teórico que permitisse um processo permanente de diálogo com a realidade do sistema de saúde brasileiro, levando em consideração na construção de seus indicadores e de suas matrizes de organização ao mesmo tempo a situação de saúde da população idosa no Brasil e a evolução das políticas públicas adotadas pelo governo para resolver problemas sociais.

Uma inovação importante é a disponibilização de indicadores a nível municipal. O papel dos municípios na formulação, acompanhamento e implementação de programas de saúde vem aumentando com os processos de descentralização e regionalização do SUS, iniciados com a NOASSUS 01 de 2001 e aprimorados pelo Pacto pela Saúde, de 2006, e depois pelo COAP, de 2011. No

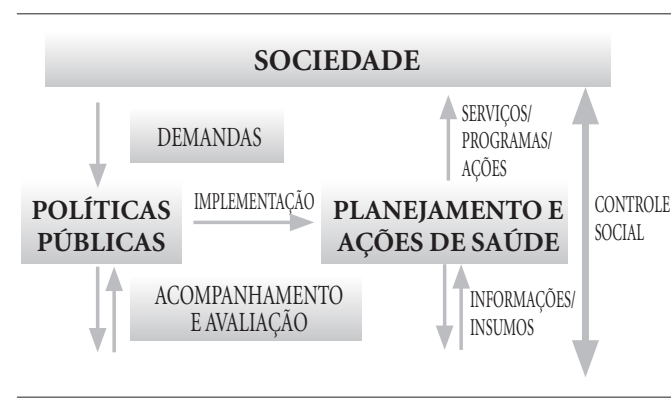

Figura 1. Proposta do SISAP-Idoso.

Fonte: elaboração própria.

entanto, muitas informações disponíveis no Sistema foram levantadas em uma escala que não permite desagregação.

O SISAP-Idoso conta com informações de diferentes fontes, pesquisas e inquéritos. Para produzir os indicadores foram utilizadas informações do SIA/SUS (Sistema de Informações Ambulatoriais do Sistema Único de Saúde), SIM (Sistema de Informações sobre Mortalidade), SI-PNI (Sistema de Informações do Programa Nacional de Imunizações), SIH/SUS (Sistema de Informações Hospitalares do Sistema Único de Saúde). As pesquisas e os inquéritos utilizados no SISAP são selecionadas de acordo com os dois critérios: (1) Devem ser representativos da população e (2) devem ser desagregáveis e representativos da população idosa de 60 anos e mais. Assim, são utilizadas no Sistema a PNAD (Pesquisa Nacional por Amostra de Domicílios), a PNS 2013 (IBGE - Pesquisa Nacional de Saúde - PNS 2013) e o VIGITEL. Informações demográficas são obtidas através dos Censos Demográficos realizados pelo IBGE.

\section{Matriz conceitual por dimensões de saúde}

$\mathrm{Na}$ Matriz Conceitual por Dimensões de Saúde do SISAP-Idoso o arcabouço teórico desenvolvido pelo PROADESS foi adaptado para as condições específicas de saúde dos idosos e seus objetivos foram revisados para que acordassem com a ideia central do SISAP-Idoso. Por este motivo, enquanto no PROADESS a matriz serve para a avaliação do sistema de saúde, no SISAP-Idoso ela é utilizada para o diagnóstico da situação de saúde da população idosa, procedimento sem o qual não é possível planejar adequadamente ações de saúde. 
A ideia por trás desta Matriz é que somente com informação adequada sobre os fatores que influenciam a situação de saúde dos idosos é possível realizar a formulação e o acompanhamento de políticas e programas de saúde. Ou seja, na perspectiva da saúde enquanto o estado de completo bem-estar físico, mental e social o acompanhamento do sucesso de políticas e programas de saúde depende de um diálogo com as condições gerais de saúde da população que pretende afetar.

Esta Matriz é composta por quatro grandes dimensões que são subdivididas em áreas. Os indicadores calculados foram alocados conforme o tema ao qual estão relacionados. Abaixo, o esquema da divisão das dimensões do SISAP-Idoso:

1. Determinantes da saúde

a. Indicadores contextuais e ambientais

b. Indicadores socioeconômicos e de

fragilidade social

c. Indicadores demográficos

d. Indicadores comportamentais

2. Condições de saúde dos idosos

a. Indicadores de bem-estar

b. Indicadores de estado funcional

c. Indicadores de morbidade

i. Mortalidade por causa

3. Serviços de saúde

a. Indicadores de efetividade

i. Causas evitáveis de mortalidade

ii. Causas evitáveis de internação

b. Indicadores de acesso

i. Cobertura vacinal

ii. Cobertura de PACS e ESF

iii. Assistência farmacêutica

iv. Direito da pessoa idosa

c. Indicadores de adequação

d. Indicadores de financiamento

4. Qualidade da informação

a. Indicadores de qualidade do SIM

b. Indicadores de qualidade do SIH/SUS

A partir das informações do Censo Demográfico, foram calculados os indicadores da primeira dimensão do sistema, a Determinantes da Saúde, dividida em subdimensões que tratam dos fatores ambientais que atuam como determinantes de agravos à saúde (Indicadores contextuais e ambientais), dos fatores econômicos e sociais da população idosa (Indicadores socioeconômicos e de fragilidade social), de características demográficas da população (Indicadores demográficos) e de fatores relacionados a comportamentos, atitudes e práticas que podem influenciar a ocorrência de doenças e agravos (Indicadores comportamentais).
A segunda dimensão traz indicadores relacionados às Condições de Saúde dos Idosos e se subdivide em: indicadores de qualidade de vida associada ao bem-estar dos idosos (Indicadores de bem-estar), de limitações no desempenho das funções corporais e da realização de atividades cotidianas típicas (Indicadores de Estado Funcional), indicadores que mostram o perfil das internações e dos óbitos na população idosa por causas (Indicadores de Morbidade e Mortalidade). Foram utilizadas informações da Pesquisa Nacional por Amostra de Domicílios (PNAD), do Censo Demográfico, do Sistema de Informações Hospitalares do SUS (SIH/SUS), do Sistema de Informações sobre Mortalidade (SIM) e da Pesquisa Nacional de Saúde (PNS).

Os indicadores que tratam dos Serviços de Saúde, terceira dimensão, foram calculados utilizando como fonte de informação o SIM, o SIH/SUS, a PNAD, o Sistema de Informações do Programa Nacional de Imunizações (SI-PNI), o Sistema de Informações Ambulatoriais do SUS (SIA-SUS) e a PNS. São subdivisões os temas: Efetividade, mostrando o grau com que a assistência, os serviços e as ações de saúde atingem os resultados esperados, compreendendo indicadores de causas evitáveis de mortalidade e internação; Acesso, com indicadores de utilização dos meios oferecidos pelo setor público para atender às necessidades de saúde da população idosa; Adequação, que indica se os cuidados oferecidos atendem aos padrões estabelecidos pelo conhecimento técnico-científico existente; e Financiamento, que são indicadores referentes às formas de financiamento dos serviços de saúde.

Por fim, a quarta dimensão, Qualidade da Informação, possui indicadores que avaliam a qualidade do preenchimento das Declarações de Óbito (DO) e das Autorizações para Internação Hospitalar (AIH) de idosos, considerando a completitude das informações do SIM e do SIH/SUS. Especificamente na subdimensão SIM (mortalidade), há indicadores de classificação quanto ao preenchimento de informações socioeconômicas e de atenção médica, e um indicador resumo combinado que considera a proporção de óbitos de idosos com causa básica mal definida (PCMD) e com causa básica não especificada (PCI). Os indicadores do sistema podem ser acessados por diversas formas de consulta, nas quais o usuário pode visualizar a informação por abrangência geográfica, por sexo e por tipo (número, proporção e taxa). 


\section{Matriz conceitual para acompanhamento de políticas e programas}

Uma das principais inovações do SISAP-Idoso dentre os sistemas de M\&A de programas de saúde existentes no Brasil é a disponibilização de uma Matriz Conceitual que permite o acompanhamento de dispositivos específicos de políticas e programas de saúde da pessoa idosa através de indicadores selecionados.

A importância deste tipo de instrumento para a gestão em saúde vem crescendo nas últimas décadas. A complexidade do SUS faz com que nossa política de saúde seja necessariamente dividida em vários componentes e ações menores, o que se intensifica devido às especificidades da população idosa. A aplicação dos mesmos programas em múltiplas localidades (com suas diferenças regionais), em múltiplos níveis de gestão (municipais, estaduais e federal) e público-alvo variado (com as diferenças de renda, gênero, rural/urbano etc.) torna essencial saber quais componentes planejados foram realmente implementados.

Uma vez que o programa foi implementado, é importante entender quais componentes contribuíram para o resultado positivo de intervenções bem-sucedidas. Quando as intervenções fracassam temos que saber quais componentes levaram ao resultado negativo.

O objetivo desta Matriz é permitir acessar os diversos estágios de programas de saúde da pessoa idosa e fornecer ferramentas capazes de captar empiricamente a medida na qual os programas estão sendo implementados e se estão tendo sucesso. Para atingir esta finalidade, no SISAP-Idoso os indicadores são vistos como ferramentas úteis na transformação do conteúdo normativo da agenda de saúde brasileira em algo concreto. Indicadores específicos são apresentados para acompanhar as medidas previstas nos programas.

A utilização de indicadores para o acompanhamento de políticas e programas é uma forma de ajudar o Estado a avaliar o seu próprio progresso. Além disso, a disponibilização de informações precisas e pertinentes ajuda os tomadores de decisão na formulação de novas políticas públicas. $\mathrm{O}$ uso de indicadores adequados agiliza o processo de tomada de decisões, torná-lo mais transparente e eficaz e reduzir o esforço na obtenção de informações. Por fim, a existência de indicadores selecionados para o acompanhamento de programas de saúde permite que as instituições da sociedade civil supervisionem a ação do Estado na promoção da saúde do idoso ${ }^{7,8}$.
Para facilitar a visualização por parte do público-alvo, principalmente de gestores, os indicadores são disponibilizados por política, divididos entre pactuados e não pactuados. Foram selecionadas políticas que tratassem diretamente da população idosa ou de agravos à saúde mais prevalentes nesse grupo etário, bem como fatores de risco associados.

\section{Critérios de definição dos aspectos monitorados e avaliados pelo SISAP}

O SISAP-Idoso trabalha com uma concepção estagista do processo de políticas públicas. Ou seja, atribui teoricamente momentos temporais distintos para a implementação de uma política ou programa e para seus resultados. Desta forma, o monitoramento da implementação e a avaliação dos resultados também serão representados em momentos definidos e separados. Como veremos a seguir, isso permitirá que o sistema estabeleça critérios específicos para cada uma das fases.

Cada um desses momentos apresenta uma multiplicidade de aspectos que podem ser analisados quando realizamos o monitoramento e a avaliação de uma política ou programa social.

No caso da implementação, o monitoramento pode conter os seguintes aspectos ${ }^{9}$ :

- Recrutamento: o quanto o programa consegue mobilizar instituições e burocracia;

- Manutenção: o quanto o programa consegue manter os participantes envolvidos;

- Contexto: o conjunto de circunstâncias institucionais relacionadas à implementação;

- Recursos: quais os recursos disponíveis para implementar o programa;

- Extensão: até que ponto o programa foi implementado como formulado (metas);

- Alcance: área coberta pelo programa (pode ser calculado com base em vários critérios, inclusive territorial);

- Barreiras: problemas encontrados em alcançar o público;

- Exposição: até que ponto informações sobre o programa chegam ao público-alvo;

- Uso inicial: como as pessoas têm acesso ao programa;

- Uso continuado: as pessoas que tiveram acesso o continuam utilizando?;

- Contaminação: a extensão na qual o público recebe intervenções de outros programas que afetam a implementação.

Já os resultados podem ser avaliados de acordo $\operatorname{com}^{10}$ :

- Eficiência: termo originado nas ciências econômicas que significa a menor relação custo/ 
benefício possível para o alcance dos objetivos estabelecidos no programa;

- Eficácia: medida do grau em que o programa atinge os seus objetivos e metas;

- Impacto (ou efetividade) - indica se o projeto tem efeitos (positivos) no ambiente externo em que foi feita a intervenção, em termos técnicos, econômicos, socioculturais, institucionais e ambientais;

- Sustentabilidade: mede a capacidade de continuidade dos efeitos benéficos alcançados através do programa social, após o seu término;

- Análise custo-efetividade: similar à ideia de custo de oportunidade e ao conceito de pertinência; é feita a comparação de formas alternativas da ação social para a obtenção de determinados impactos, para ser selecionada aquela atividade/ projeto que atenda os objetivos com o menor custo;

- Satisfação do beneficiário: avalia a atitude do usuário em relação à qualidade do atendimento que está obtendo do programa;

- Equidade: procura avaliar o grau em que os benefícios de um programa estão sendo distribuídos de maneira justa e compatível com as necessidades do usuário.

A opção por formular um sistema amplo de acompanhamento através de indicadores limita a escolha de quais dos componentes acima farão parte da Matriz Conceitual. Um dos principais problemas encontrados é a disponibilidade de dados. Nesse contexto, os aspectos selecionados para monitoramento no SISAP-Idoso são: (i) recursos (humanos, de infraestrutura e orçamentários) disponíveis para a implementação do programa e (ii) da extensão na qual os recursos disponíveis são aplicados. No que tange à avaliação de resultados, o Sistema tem seu enfoque na eficácia, em medir o grau em que o programa atinge os seus objetivos e metas.

Para o acompanhamento de políticas e programas de saúde do idoso em termos da eficiência no uso dos recursos, da eficácia no cumprimento de metas e da efetividade dos seus desdobramentos sociais mais abrangentes e perenes, buscam-se dados administrativos (gerados no âmbito dos programas ou em outros cadastros oficiais) e estatísticas públicas (produzidas pelo IBGE e outras instituições). Esses dados são reorganizados na forma de taxas, proporções, índices ou mesmo em valores absolutos e transformamse em indicadores sociais.

Contudo, é impossível colher informações sobre todos os aspectos de uma determinada realidade social. Assim, os Sistemas de Informação contam com alguns indicadores selecionados que não cobrem todos os aspectos monitoráveis e avaliáveis listados acima.

Os indicadores disponíveis no SISAP-Idoso estão sujeitos às rotinas de alimentação de suas respectivas fontes de dados (como SIM, SIH, etc.). Os inquéritos populacionais, por exemplo, são disponibilizados com certa defasagem em relação ao tempo de coleta. Assim, os indicadores do Sistema ficam sujeitos ao fluxo de processamento de dados dos Sistemas de informação em Saúde do SUS, tornando a periodicidade de atualização do SISAP-Idoso em desafio.

\section{Organização e critérios para a seleção de indicadores}

A maneira mais eficiente de permitir que a avaliação de programas e políticas de saúde possa contribuir para testar a consistência do planejamento e o alcance dos resultados esperados é a definição prévia da matriz lógica do programa ${ }^{11}$.

A construção da matriz lógica começa pela identificação dos objetivos gerais e específicos do programa e das atividades ou açoes a eles correlacionados. Em um segundo momento, são indicadas as fontes de informação disponíveis para realizar as mensurações, comparações e análises necessárias. Posteriormente, são identificados os recursos (financeiros, humanos e de infraestrutura) alocados ao programa, as atividades previstas, os resultados esperados e as relações causais (uma particular combinação de recursos, permitindo a realização de um determinado conjunto de atividades, que levará à produção de determinados resultados, que, presume-se, permitirão que se alcancem determinados objetivos associados ao programa) que a teoria por trás do programa pressupõe que podem ser assumidas. Por último, são definidos os indicadores de performance pelos quais os resultados do programa serão avaliados (Figura 2).

Os indicadores para identificação dos recursos alocados servem para monitoramento da implementação do programa ou política de saúde do idoso. Os indicadores para avaliação de resultados constituem a variante clássica de avaliação e servem para medir o alcance de metas e os efeitos de políticas e programas.

Os indicadores selecionados para cada uma das partes podem ter diversas origens. Eles podem ser absolutos, quando o próprio programa estabelece as metas a serem alcançadas e elas são consideradas o padrão. Podem também ser negociados ou de compromisso, quando se baseiam 
Figura 2. Construção de Matriz Lógica para Ciclo de Políticas Públicas.

Fonte: elaboração própria.

em algum procedimento específico para sua fixação, geralmente decorrente de acordo entre as partes envolvidas na gestão de programa e os formuladores. Sempre que disponíveis estes indicadores devem ser utilizados.

No caso de o programa não determinar indicadores e não haver pacto, os indicadores para acompanhamento podem ser históricos, comparam resultados de um período com o obtido em anteriores; normativos, comparam o desempenho de um programa com outros similares ou com semelhantes realizados em outros níveis de governo, região, ou no exterior; ou teóricos, são os estabelecidos na própria elaboração do programa, sob a hipótese da obtenção dos resultados esperados, dado os recursos disponíveis.

Quando não existem metas pactuadas ou definidas pela própria política, a escolha de indicadores deve ser pautada por sua aderência a um conjunto de propriedades desejáveis ${ }^{12}$. A primeira propriedade fundamental para a escolha do indicador é a validade, pois é desejável que se disponha de medidas tão próximas quanto possível do conceito abstrato ou demanda política que deram origem à política.

A confiabilidade da medida é outra propriedade essencial para legitimar o uso do indicador. Sempre que possível, deve-se procurar também empregar indicadores com cobertura territorial ou populacional adequada, que sejam representativos da realidade em análise.

A sensibilidade e a especificidade também devem ser avaliadas na escolha do indicador. É importante dispor de medidas sensíveis e específicas às ações previstas no programa, que possibilitem avaliar rapidamente os efeitos (positivos ou negativos) de determinada intervenção.

A periodicidade com que o indicador pode ser atualizado e a factibilidade de sua obtenção são outros aspectos cruciais. É necessário dispor de indicadores que possam ser usados em tempo hábil para monitorar uma política e seus resultados e reconfigurá-la no caso de um problema. Por isso, deve-se buscar indicadores que usem dados acessíveis e regularmente atualizados.

Para o SISAP-Idoso é essencial que os indicadores se refiram, tanto quanto possível, à população-alvo do Sistema. Indicadores que tratem diretamente da população idosa devem ser preferidos àqueles que tratem da população em geral. Por último, a possibilidade de construção de uma série histórica também é uma característica desejável porque permite a inferência de tendências e a avaliação dos efeitos dos programas implementados.

Na prática, nem todos os indicadores apresentarão todos os critérios desejáveis. O importante é que a escolha dos indicadores seja fundamentada na avaliação crítica das propriedades discutidas ${ }^{13}$.

\section{Taxonomia dos indicadores para fins de aplicação no monitoramento e avaliação da política de saúde e proposta do SISAP-Idoso}

Cada fase do processo de formulação e implementação da política de saúde do idoso requer, portanto, o emprego de indicadores específicos, cada qual trazendo elementos e subsídios distintos para o bom encaminhamento do processo.

A lógica avaliativa do SISAP-Idoso busca apreender, de maneira simplificada, as estrutu- 
ras, as propriedades e os atributos funcionais do SUS. Para isto, são incluídos no modelo sistêmico itens básicos como os recursos, os processos e os produtos necessários e esperados para uma bemsucedida ação de saúde.

Os indicadores-insumo correspondem às medidas associadas à disponibilidade de recursos humanos, financeiros ou equipamentos alocados para um processo ou programa que afeta uma das dimensões da realidade social. São tipicamente indicadores de alocação de recursos para políticas sociais o número de leitos hospitalares por mil habitantes, o número de professores por quantidade de estudantes ou ainda o gasto monetário per capita nas diversas áreas de política social.

Os indicadores-produto são aqueles mais propriamente vinculados às dimensões empíricas da realidade social, referidos às variáveis resultantes de processos sociais complexos, como a esperança de vida ao nascer, a proporção de crianças fora da escola ou o nível de pobreza. São medidas representativas das condições de vida, saúde, nível de renda da população, indicativas da presença, ausência, avanços ou retrocessos das políticas sociais formuladas. Enquanto os indicadores-insumo quantificam os recursos disponibilizados nas diversas políticas sociais, os indicadores-produto retratam os resultados efetivos dessas políticas.

Os indicadores-processo ou fluxo são indicadores intermediários, que traduzem em medidas quantitativas o esforço operacional de alocação de recursos humanos, físicos ou financeiros (indicadores-insumo) para a obtenção de melhorias efetivas de bem-estar (indicadores-produto), como número de consultas pediátricas por mês, merendas escolares distribuídas diariamente por aluno, ou ainda homens-hora dedicados a um programa social.

Em alguns contextos, os indicadores de insumo e processo costumam ser chamados de indicadores de esforço, e os indicadores-produto de indicadores de resultados. Além disso, alguns autores destacam ainda que alguns dos resultados podem ser classificados como o impacto geral do programa, por se tratarem de desdobramentos mais gerais, a serem alcançados no médio prazo, ou ainda por se tratarem de externalidades positivas ou mesmo não antecipadas de determinado programa.

$\mathrm{Na}$ implementação dos programas é preciso avaliar o esforço despendido através de indicadores-insumo e indicadores-processo de avaliação da eficiência dos recursos empregados. Já na avaliação dos programas implementados, os resultados devem ser aferidos através de indicadores-produtos de diferentes tipos para medir a eficácia no cumprimento das metas específicas e a efetividade social das soluções sugeridas. Uma política municipal de saúde, por exemplo, deve ser avaliada através do esforço na dotação de recursos para a área, de como estes recursos são usados e como a política contribui para a melhoria das condições de saúde e de vida da população $0^{14}$.

Vale lembrar que ao contrário de outras louváveis experiências de M\&A do sistema de saúde - como, por exemplo, o Modelo Teórico do Painel de Monitoramento e Avaliação da Gestão do SUS, desenvolvido a partir de uma parceria entre Abrasco, DEMAGS e SGEP/MS ${ }^{15}$, ou o PROADESS, desenvolvido pela Fiocruz - o SISAP-Idoso não está voltado para o monitoramento e avaliação do sistema de saúde e sim de dispositivos de políticas e programas que afetem a saúde do idoso.

Seu objetivo não é, portanto, avaliar se o SUS está cumprindo seus objetivos, mas se determinados dispositivos de programas de saúde estão sendo implementados e alcançando os resultados esperados. Mais do que realizar uma avaliação de desempenho do sistema de saúde, o SISAP-Idoso pretende servir como instrumento de planejamento e acompanhamento de políticas públicas específicas para a população idosa.

Abaixo um exemplo da aplicação da Matriz de M\&A do SISAP-Idoso. A Política Nacional de Saúde da Pessoa Idosa é composta de nove diretrizes (objetivos específicos) com diversas medidas previstas para sua implementação, no Quadro 1 apresentamos o esquema de M\&A de algumas medidas previstas na Diretriz 3.1 "Promoção do Envelhecimento Ativo e Saudável”.

\section{Considerações finais}

Embora o SISAP-Idoso apresente contribuições significativas para a gestão em saúde do idoso, muitos desafios permanecem. O Sistema atinge seu objetivo de disponibilizar informações para o planejamento e o acompanhamento de políticas e programas de saúde do idoso. No entanto, a formulação de políticas sem diretrizes e metas devidamente explicitadas se mostra uma barreira para o uso de indicadores na gestão em saúde.

Outro fator que prejudica a utilização do Sistema é a falta de informações sensíveis para o acompanhamento das políticas. Por vezes, os indicadores utilizados representam apenas parte do 
Quadro 1. Painel de Indicadores para Monitoramento e Avaliação da Diretriz 3.1 da Política Nacional de Saúde da Pessoa Idosa.

\begin{tabular}{|c|c|c|c|}
\hline \multicolumn{4}{|c|}{ Política Nacional de Saúde da Pessoa Idosa } \\
\hline Objetivo Geral & \multicolumn{3}{|c|}{$\begin{array}{l}\text { A finalidade primordial da Política Nacional de Saúde da Pessoa Idosa } \\
\text { é recuperar, manter e promover a autonomia e a independência dos } \\
\text { indivíduos idosos, direcionando medidas coletivas e individuais de } \\
\text { saúde para esse fim, em consonância com os princípios e diretrizes do } \\
\text { Sistema Único de Saúde. É alvo dessa política todo cidadão e cidadã } \\
\text { brasileiros com } 60 \text { anos ou mais de idade. }\end{array}$} \\
\hline Objetivo Específico & \multicolumn{3}{|c|}{ 3.1. Promoção do Envelhecimento Ativo e Saudável } \\
\hline \multirow[t]{2}{*}{ MEDIDA } & $\begin{array}{l}\text { INDICADORES DE } \\
\text { IMPLEMENTAÇÃO }\end{array}$ & & $\begin{array}{c}\text { INDICADORES DE } \\
\text { RESULTADO }\end{array}$ \\
\hline & INSUMO & PROCESSO & PRODUTO \\
\hline $\begin{array}{l}\text { n) promover a saúde por meio de } \\
\text { serviços preventivos primários, tais } \\
\text { como a vacinação da população } \\
\text { idosa, em conformidade com a } \\
\text { Política Nacional de Imunização; }\end{array}$ & $\begin{array}{l}\text { Proporção de } \\
\text { estabelecimentos de } \\
\text { saúde participantes } \\
\text { da campanha de } \\
\text { vacinação contra } \\
\text { gripe }\end{array}$ & $\begin{array}{l}\text { Cobertura vacinal } \\
\text { de idosos contra } \\
\text { gripe }\end{array}$ & $\begin{array}{l}\text { Taxa de Internação de } \\
\text { Idosos para tratamento } \\
\text { de pneumonia e gripe } \\
\text { consideradas evitáveis } \\
\text { Taxa de mortalidade de } \\
\text { idosos por pneumonia e } \\
\text { gripe consideradas evitáveis }\end{array}$ \\
\hline $\begin{array}{l}\text { r) implementar ações para reduzir } \\
\text { hospitalizações e aumentar } \\
\text { habilidades para o auto-cuidado } \\
\text { dos usuários do SUS; }\end{array}$ & $\begin{array}{l}\text { Cobertura da } \\
\text { Estratégia de Saúde } \\
\text { da Família }\end{array}$ & $\begin{array}{l}\text { Proporção de } \\
\text { idosos inscritos } \\
\text { na Estratégia de } \\
\text { Saúde da Família }\end{array}$ & $\begin{array}{l}\text { Taxa de internações de } \\
\text { idosos consideradas } \\
\text { evitáveis por atenção } \\
\text { primária }\end{array}$ \\
\hline
\end{tabular}

Fonte: elaboração própria.

que se quer monitorar ou avaliar. Ainda assim, dentre as possibilidades disponíveis foram selecionados os indicadores que melhor representavam a situação investigada.

Para que seja possível adequar os indicadores do SISAP-Idoso às demandas da gestão em saúde do idoso é importante também estimular e reforçar a relevância das informações em saúde e especialmente da qualidade das mesmas. $\mathrm{O}$ registro das atividades em saúde é essencial para a produção de informações de qualidade. Cabe então sensibilizar os profissionais para o devido preenchimento das fontes de dados.

Como perspectiva, a incorporação plena do SISAP-Idoso depende da consolidação do uso de informações e indicadores nos processos de monitoramento e avaliação de políticas e programas de saúde. Para tal, a capacitação dos gestores para o desempenho dessas atividades se mostra uma iniciativa fundamental. Além disso, deve-se estabelecer uma cultura avaliativa no Sistema Único de Saúde. Um acompanhamento contínuo das condições de saúde em todos os níveis de gestão permite o planejamento de ações que visem o desenvolvimento e o fortalecimento do SUS.
O SISAP-Idoso, além de poder ser uma ferramenta utilizada por gestores de saúde e acadêmicos se mostra como uma poderosa ferramenta de controle social e empoderamento da população, na medida que permite o conhecimento da situação e saúde e a identificação e a inclusão de assuntos na agenda de prioridades.

\section{Colaboradores}

DE Romero e AP Marques trabalharam na concepção e redação final, D Castanheira trabalhou na metodologia e na redação final, J Muzy e L Sabbadini trabalharam na redação final e na produção do material ilustrativo e RS Silva trabalhou na metodologia do artigo. 


\section{Referências}

1. Brasil. Ministério da Saúde (MS). Considerações sobre a instituição de um processo de monitoramento e avaliação do SUS. Brasília: MS; 2005. [Nota Técnica].

2. Felisberto E. Da teoria à formulação de uma Política Nacional de Avaliação em Saúde: reabrindo o debate. Cien Saude Colet 2006; 11(3):553-563.

3. Cohn A, Westphal MF, Elias PE. Informação e decisão política em saúde. Rev Saude Publica 2005; 39(1):114121.

4. Paim JS. Editorial. Tempos de Avaliação em Saúde. Revista de Ciências Médicas e Biológicas 2005; 4(1):3-6.

5. Viacava F, Ugá MA, Porto S, Laguardia J, Moreira RS. Avaliação de Desempenho de Sistemas de Saúde: um modelo de análise. Cien Saude Colet 2012; 17(4):921934.

6. Maia Diniz I, Guerra Júnior AA, Lovato Pires Lemos L, Souza KM, Godman B, Bennie M, Wettermark B, Assis Acurcio F, Alvares J, Gurgel Andrade EI, Leal Cherchiglia M, Araújo VE. Uma metodologia de avaliação do desempenho do sistema de saúde brasileiro. Cien Saude Colet 2004; 9(3):711-724.

7. Assis MMA, Villa TCS. O controle social e a democratização da informação: um processo em construção. Rev Latino-am. Enfermagem 2003; 11(3):376-382.

8. Silva AX, Cruz EA, Melo V. A importância estratégica da informação em saúde para o exercício do controle social. Cien Saude Colet 2007; 12(3):683-688.

9. Steckler A, Linnan L. Process evaluation for public health interventions and research. An Overview. In: Steckler A, Linnan L, editors. Process evaluation for public health interventions and research. San Francisco: Jossey Bass; 2002. p. 9-23.

10. Costa FL, Castanhar JC. Avaliação de programas públicos: desafios conceituais e metodológicos. Revista de Administração Pública 2003; 37(5):962-969.

11. Wholey JS. Assessing the feasibility and likely usefulness of evaluation. In: Wholey JS, Hatry HP, Newcomer KE, editors. Handbook of practical program evaluation. $3^{\text {rd }}$ ed. San Francisco: Jossey-Bass Publishers; 2010. p. 33-62.

12. Jannuzzi PM. Indicadores para diagnóstico, monitoramento e avaliação de programas sociais no Brasil. Revista do Serviço Público 2005; 56(2):137-60.

13. Jannuzzi PM. Indicadores para diagnóstico, monitoramento e avaliação de programas sociais no Brasil. Revista do Serviço Público 2005; 56(2):137-160.

14. Jannuzzi PM. Indicadores sociais na formulação e avaliação de políticas públicas. Revista Brasileira de Administração Pública 2002; 36(1):51-72.

15. Tamaki EM, Tanaka OY, Felisberto L, Alves CKAA, Drumond Júnior M, Bezerra LCA, Calvo MCM, Miranda AS. Metodologia de construção de um painel de indicadores para o monitoramento e a avaliação da gestão do SUS. Cien Saude Colet 2012; (4):839-849. 